\title{
Manual and electronic probes have similar reliability in the measurement of untreated periodontitis
}

\author{
Abstracted from \\ Silva-Boghossian CM, Amaral CS, Maia LC, Luiz RR, Colombo AP. \\ Manual and electronic probing of the periodontal attachment level in untreated periodontitis: \\ a systematic review. J Dent 2008; 36: 651-657 \\ Address for correspondence: Carina Silva-Boghossian, Rua Barão de Guaratiba, 228 Glória, Rio de Janeiro, \\ RJ, Cep 22211-150, Brazil. E-mail address: carinabogho@hotmail.com
}

\section{Question: Do manual or electronic probes produce the most reproducible measurements of clinical attachment level in periodontitis patients?}

Data Sources The Cochrane Central Register of Controlled Trials, PubMed Medline and Latin American and Caribbean Health Science literature databases were searched, and searches made by hand of identified papers, to source relevant data. (BIOSIS Previews) were searched. There were no language restrictions.

Study selection Studies were evaluated by two reviewers independently. Only clinical trials were included that were published in the English, Spanish and Portuguese languages and were performed on humans. Articles were excluded when the examiners were not calibrated; when they did not compare manual and electronic probing techniques; when they did not measure the clinical attachment level (CAL); and when the subjects did not present destructive periodontal disease or had already received periodontal treatment.

Data extraction and synthesis The quality of the identified studies was assessed and standardised data extracted. Only two studies met all the selection criteria so no meta-analysis was performed.

Results Only two of the 37 identified articles were included in the review. The results of these two studies showed that the mean variance and the absolute mean difference between CAL measurements for the two types of probes were not statistically different.

Conclusions Manual and electronic probes were of similar reliability when used to measure CAL in individuals who had untreated periodontitis and when used by a calibrated examiner, but this finding is not supported by strong evidence.

\section{Commentary}

It is the sailor not the song! The results of this well-executed systematic review, like the siren's songs encountered by Ulysses in Homer's Odyssey, are captivating.

Clinicians have been led to believe that each of the two types of periodontal probe - manual and electronic — has alluring mantic 'truths'. The manual periodontal probe has the advantage of ease of use, easy accessibility and low cost. The electronic probe is equally attractive with its automated electronic recording, controlled force and accurate measurements. Ulysses was not led astray by the siren's songs and reliably sailed to Ithaca, returning to the same seaport from which he had departed on his journey. Less reliable is the dentist, arriving at an identical valid CAL. Hence, the development and presumed appeal of electronic probes.

The variation in manual probe reliability and validity was documented almost 25 years ago. ${ }^{1,2}$ Using calibrated clinicians, the theoretical and clinical findings were, first, that the standard error in probe measurements is approx. $1 \mathrm{~mm}$ (following repeated measurements by the same calibrated clinician in the same subject). ${ }^{1}$ Second, the minimum significant change that can reliably be detected is about $2 \mathrm{~mm} .^{2}$ In other words, accurate probe measurement [ie, reduction of false positives (such as periodontal disease presence or progression when there is none) and false negatives (periodontal health when there is disease or disease progression)] requires calibrated examiners and duplicate measures when using a manual probe.

Navigating the waters off Ionia may have been treacherous for Ulysses: navigating the periodontal pocket can be equally treacherous for the cautious clinician. Based on the reliable measurement of clinical attachment, the identified and reviewed trials indicate that either probe, when used by calibrated clinicians, is equally reliable. The current review again makes clear that it is the cautious sailor, not the siren song, that best guides the ship of care.

Richard Niederman,

Forsyth Institute, Boston, Massachusetts, USA

Practice points

- For calibrated clinicians, manual and electronic probes are equally reliable in measuring periodontal pockets.

1. Goodson JM. Clinical measurements of periodontitis. J Clin Periodontol 1986; 13: $446-460$.

2. Haffajee AD, Socransky SS. Attachment level changes in destructive periodontal diseases. / Clin Periodontol 1986; 13: 461-475.

Evidence-Based Dentistry (2009) 10, 39. doi:10.1038/sj.ebd.6400643 